\title{
Diagrama de fases experimental Fe-Sn-Zr. Nuevos resultados del corte isotérmico de $800^{\circ} \mathrm{C}$
}

\author{
Nicolás Nieva ${ }^{1}$, Martín Rodrigo Tolosa ${ }^{1}$, \\ Carolina Corvalán ${ }^{2}$, Delia Arias ${ }^{3}$
}

\begin{abstract}
1 Laboratorio de Física del Sólido, Departamento de Física, Facultad de Ciencias Exactas y Tecnología, Universidad Nacional de Tucumán, Av. Independencia 1800, San Miguel de Tucumán, Tucumán, Argentina. e-mail: nnieva@herrera.unt.edu.ar 2 Gerencia de Materiales, Comisión Nacional de Energía Atómica Argentina. Universidad Nacional de Tres de Febrero. CONICET, Consejo Nacional de Ciencia y Técnica, Buenos Aires, Buenos Aires, Argentina.

3 Instituto de Tecnología J. Sábato, Comisión Nacional de Energía Atómica Argentina. Universidad Nacional de San Martín, San Martín, Buenos Aires, Argentina.
\end{abstract}

\section{RESUMEN}

Las aleaciones de base $\mathrm{Zr}$ son de extendida aplicación en el campo de la tecnología nuclear. El Zr aleado con Sn y Fe es la base principal de las aleaciones tipo Zircaloy, aún hoy muy utilizadas como elementos estructurales y como contenedores de los elementos combustibles en reactores nucleares de potencia. Este sistema metalúrgico ternario también forma parte de aleaciones de más reciente desarrollo como las Zirlo (EEUU). Si bien en todas ellas el $\mathrm{Zr}$ es el aleante mayoritario es útil e importante conocer los diagramas de fases de sus componentes en la forma más completa posible.

El diagrama de fases $\mathrm{Fe}-\mathrm{Sn}-\mathrm{Zr}$ viene siendo estudiado en forma sistemática por el grupo de trabajo desde hace años. Se ha avanzado mucho en el trazado del diagrama de fases a $800^{\circ} \mathrm{C}$, pero han quedado regiones del triángulo de Gibbs sin resolver. Con el objeto de evaluar experimentalmente estas zonas se procedió al diseño y fabricación de un conjunto de aleaciones ternarias. Las mismas fueron tratadas térmicamente a la temperatura de interés por 2928 horas y caracterizadas aplicando técnicas de metalografía, difracción de rayos $\mathrm{X}$ y de microanálisis. En este trabajo se confirmó la existencia de los compuestos ternarios $\mathrm{Y}$ y $\mathrm{Fe}_{6} \mathrm{Sn}_{6} \mathrm{Zr}$, así como la presencia de la fase X" y se determinaron campos de existencia de 2 y 3 fases, todo a $800^{\circ} \mathrm{C}$. Finalmente se trazaron los límites de equilibrio de fases en las regiones bajo estudio, completando en gran proporción el diagrama de fases para el corte isotérmico analizado.

Palabras clave: Materiales nucleares, Diagramas de Fases, Circonio.

\section{ABSTRACT}

The Zr-based alloys are extensively applied in the field of nuclear technology. Alloyed with $\mathrm{Sn}$ and $\mathrm{Fe}, \mathrm{Zr}$ is the main element in the zircaloy-type alloys, currently vastly used as structural elements and as containers of burnable elements in nuclear reactors. This ternary metallurgical system is also a component of more recently developed alloys such as Zirlo (USA). Although $\mathrm{Zr}$ is a major component in all of them, it is most important to know the phase diagrams of their components as best as possible. The Fe-Sn-Zr phase diagram has been systematically studied by our research team for many years. Considerable advancement has been made in the design of the phase diagram at $800^{\circ} \mathrm{C}$, however, some regions in the Gibbs triangle are still unsolved. In order to evaluate these regions experimentally, a ternary alloy set was designed and prepared. These alloys were thermally treated at the chosen temperature for 2928 hours, and characterized by applying metallography techniques, X-ray diffraction and microanalysis. The presence of $\mathrm{Y}$ and $\mathrm{Fe}_{6} \mathrm{Sn}_{6} \mathrm{Zr}$ was confirmed, the presence of phase X" was verified, and 2- and 3-phase existence fields were determined. Finally, phase balance boundaries in the regions under study were traced, completing, to a great extent, the phase diagram for the isothermal cut analysed.

Keywords: Nuclear materials, Phase diagram, Zirconium.

\section{INTRODUCCIÓN}


Las aleaciones de base circonio ( $\mathrm{Zr}$ ) son de extendida aplicación en el campo de la tecnología nuclear.

$\mathrm{El} \mathrm{Zr}$, aleado con estaño $(\mathrm{Sn})$ y hierro $(\mathrm{Fe})$ es la base principal de las aleaciones tipo Zircaloy, que aún hoy se emplean principalmente en la fabricación de vainas para los elementos combustibles y como materiales estructurales en los reactores nucleares de agua liviana o pesada. Esto se debe a sus características específicas: alta resistencia a la corrosión, buenas propiedades mecánicas, resistencia a daños por radiación y baja sección eficaz de captura de neutrones térmicos.

En el desarrollo de materiales aptos para la industria nuclear la seguridad es un factor clave, por las funciones críticas que se le asignan al material. Un modo esencial de abordar este aspecto es lograr un conocimiento lo más completo posible, tanto de la metalurgia física de los materiales como de los procesos metalúrgicos involucrados en la elaboración final del producto tecnológico. Por ello es que para una correcta, segura y eficiente utilización del producto, un aporte muy importante es conocer los diagramas de fases de los sistemas metalúrgicos que constituyen los elementos de partida.

El sistema ternario Fe-Sn-Zr tiene contados antecedentes de estudio de sus diagramas de fases. Entre los últimos trabajos publicados al respecto pueden mencionarse: una revisión del año 2009 realizada por Liu y col. [1], basada entre otros en los trabajos de Nieva y Arias [2,3]; el trabajo de Savidan y col. [4] que estudiaron una gran extensión del diagrama de fases de este sistema a la temperatura de $900^{\circ} \mathrm{C}$ (basados esencialmente en el trabajo de Nieva y Arias [3]). Otra recopilación realizada por Raghavan en 2011[5] está basada entre otros en los trabajos de Nieva y Arias [2,3,6] y de Savidan y col. [4]. En 2012 Nieva y col. presentaron una nueva propuesta para el corte isotérmico a $800^{\circ} \mathrm{C}$ del sistema ternario [7]. Finalmente, en 2014 Rebellato y col [8] abarcaron con su estudio la región central y adyacente al binario Fe-Sn del triángulo de Gibbs.

Si bien se ha avanzado mucho en el trazado del diagrama de fases a $800^{\circ} \mathrm{C}$, el mismo registra todavía algunas zonas sin resolver totalmente. Con la intención de completar y dar continuidad a las investigaciones sobre el diagrama de fases de este sistema, en el presente trabajo se procedió al diseño y fabricación de un grupo de aleaciones ternarias que fueron tratadas térmicamente a la temperatura de $800^{\circ} \mathrm{C}$ y caracterizadas mediante diferentes técnicas experimentales para proponer el diagrama de fases de la zona de interés.

\section{MATERIALES Y MÉTODOS}

Para la realización del trabajo experimental se diseñaron y fabricaron un conjunto de aleaciones ternarias tratando de abarcar una porción desconocida del diagrama en el triángulo de Gibbs. A las mismas se les practicó un tratamiento térmico prolongado a la temperatura de interés y se caracterizaron por medio de técnicas metalográficas (MO, microscopio óptico Olympus BX-60M), de difracción de rayos X (DRX, difractómetro de polvos, Panalytical modelo Empyrean), con microscopio electrónico de barrido con espectómetro de dispersión en energía (MEB, Zeiss Supra 55VP, EDS INCA wave 250) y con microsonda analítica electrónica (MAE, Microsonda CAMECA SXFive-WL). Para la identificación de las fases cristalinas y determinación de parámetro de red por medio de DRX se empleó el programa PowderCell 2.3.

\subsection{Fabricación de las aleaciones}

Los materiales de partida empleados para la fabricación de las muestras fueron: Fe Aldrich (pureza 99,98\%), Sn (pureza 99,999 \%) y Zr Wah-Chang (pureza 99,9\%), porcentajes expresados en peso.

Los materiales, debidamente preparados, se fundieron en un horno de arco con electrodo de tungsteno no consumible y crisol de cobre refrigerado con agua, en atmósfera con presión de argón (Ar) de alta pureza $(99,999 \%)$. Para mejorar la homogeneidad de la aleación se fundieron varias veces las muestras obteniéndose finalmente un botón de aproximadamente 8 gr. La composición nominal en porcentajes atómicos y denominación de las muestras se indican en la tabla 1.

Tabla 1: Composición nominal de las aleaciones fabricadas.

\begin{tabular}{c|c|c|c}
\hline DENOMINACIÓN & Fe (\%AT) & Sn (\%AT) & Zr (\%AT) \\
\hline T1 & 42,00 & 55,00 & 3,00 \\
\hline T2 & 55,00 & 21,50 & 23,50 \\
\hline T3 & 19,00 & 47,50 & 33,50 \\
\hline T4 & 18,90 & 43,70 & 37,40 \\
\hline T5 & 67,00 & 9,00 & 24,00 \\
\hline T6 & 66,00 & 15,00 & 19,00 \\
\hline
\end{tabular}




\subsection{Tratamiento Térmico}

Las muestras se sometieron a un tratamiento térmico de $800^{\circ} \mathrm{C}$ durante 2928 horas en un horno tubular con control de temperatura de $\pm 2{ }^{\circ} \mathrm{C}$. Previo al ingreso al horno las muestras fueron cuidadosamente limpiadas, envueltas en láminas de tantalio y encapsuladas en un tubo de vidrio de sílice, adecuadamente limpio y seco. Se purgó el tubo con Ar de alta pureza y se selló manteniendo un valor de presión interna de gas argón de $220 \mathrm{~mm}$ de $\mathrm{Hg}$. Transcurrido el tiempo fijado, el tubo fue retirado del horno e inmediatamente templado en agua sin rotura del sello.

\subsection{Preparación de la muestras}

Una vez finalizado el tratamiento térmico, se acondicionaron las muestras para su caracterización. Para el análisis de DRX se produjeron finos polvos de la muestra masiva con lima diamantada. Para la inspección metalográfica y microanálisis con MO, MEB y MAE las muestras se incluyeron en resina, se desbastaron con papel de carburo de silicio y finalmente se pulieron con paño y pasta de diamante hasta $0,25 \mu \mathrm{m}$.

\section{RESULTADOS}

La tabla 2 muestra las fases identificadas en las diferentes muestras tratadas térmicamente a $800{ }^{\circ} \mathrm{C}$, con su estructura cristalina, parámetros de red y la composición química medida con EDS y MAE.

Tabla 2: Fases principales identificadas en las muestras, con DRX, EDS y MAE. Se indican estructura cristalina, parámetros de red y composición.

\begin{tabular}{|c|c|c|c|c|c|c|c|}
\hline \multirow{3}{*}{ Muestra } & \multicolumn{4}{|c|}{ Fases Identificadas } & \multicolumn{3}{|c|}{ Composición(\%at) } \\
\hline & \multirow{2}{*}{ Fase } & \multicolumn{3}{|c|}{ Estructura cristalina } & \multirow{2}{*}{$\mathrm{Fe}$} & \multirow{2}{*}{ Sn } & \multirow{2}{*}{$\mathrm{Zr}$} \\
\hline & & Simetría & Tipo & Parámetros de red $[\AA]$ & & & \\
\hline \multirow{3}{*}{$\mathrm{T} 1$} & $\mathrm{Fe}_{6} \mathrm{Sn}_{6} \mathrm{Zr}$ & Hexagonal & $\mathrm{MgFe}_{6} \mathrm{Ge}_{6}$ & $a=b=5,318 \quad c=8,873$ & 49,3 & 45,8 & 4,9 \\
\hline & $\mathrm{Fe}_{5} \mathrm{Sn}_{3}$ & Hexagonal & $\mathrm{Co}_{1,75} \mathrm{Ge}$ & $\mathrm{a}=\mathrm{b}=4,226 \quad \mathrm{c}=5,235$ & 63,0 & 37,0 & 0,0 \\
\hline & $\beta \mathrm{Sn}$ & Cúbica (Tetragonal) & $\beta S n$ & $a=b=5,832 \quad c=3,185$ & 0,0 & 100,0 & 0,0 \\
\hline \multirow{3}{*}{$\mathrm{T} 2$} & $\mathrm{ZrFe}_{2}(\mathrm{C} 15)$ & Cúbica & $\mathrm{MgCu}_{2}$ & $\mathrm{a}=\mathrm{b}=\mathrm{c}=7,075$ & 63,7 & 12,3 & 24,0 \\
\hline & (C36) & Hexagonal & $\mathrm{MgNi}_{2}$ & $\mathrm{a}=\mathrm{b}=4,995 \quad \mathrm{c}=16,240$ & 66,0 & 15,7 & 18,3 \\
\hline & Fase $\mathrm{N}$ & Ortorrómbica & $\mathrm{Sc}_{3} \mathrm{Mg}_{2} \mathrm{Ga}_{6}$ & $a=8,167 b=8,940 c=10,726$ & 37,1 & 35,2 & 27,7 \\
\hline \multirow{3}{*}{$\mathrm{T} 3$} & Fase $\mathrm{N}$ & Ortorrómbica & $\mathrm{Sc}_{3} \mathrm{Mg}_{2} \mathrm{Ga}_{6}$ & $a=8,169 \quad b=8,964 \quad c=10,750$ & \multirow{3}{*}{\multicolumn{3}{|c|}{ n.d. }} \\
\hline & $\mathrm{ZrSn}_{2}$ & Ortorrómbica & $\mathrm{TiSi}_{2}$ & $a=5,650 \quad b=9,598 c=9,935$ & & & \\
\hline & Fase X” & n. d. & n. d. & n. d. & & & \\
\hline \multirow{3}{*}{$\mathrm{T} 4$} & Fase $\mathrm{N}$ & Ortorrómbica & $\mathrm{Sc}_{3} \mathrm{Mg}_{2} \mathrm{Ga}_{6}$ & $a=8,169 \quad b=8,964 \quad c=10,750$ & 37,0 & 35,8 & 27,2 \\
\hline & $\mathrm{ZrSn}_{2}$ & Ortorrómbica & $\mathrm{TiSi}_{2}$ & $a=5,650 \quad b=9,598 \quad c=9,935$ & 0,0 & 67,3 & 32,7 \\
\hline & Fase X” & n. d. & n. d. & n. d. & 20,0 & 41,7 & 38,3 \\
\hline $\mathrm{T} 5$ & $\mathrm{ZrFe}_{2}(\mathrm{C} 15)$ & Cúbica & $\mathrm{MgCu}_{2}$ & $\mathrm{a}=\mathrm{b}=\mathrm{c}=7,047$ & 67,2 & 6,8 & 26,0 \\
\hline T6 & (C36) & Hexagonal & $\mathrm{MgNi}_{2}$ & $a=b=4,982 c=16,207$ & 66,9 & 15,0 & 18,1 \\
\hline
\end{tabular}

\section{DISCUSIÓN}

En la muestra T1 tratada térmicamente (TT) con técnicas de DRX se identificaron cuatro fases cristalinas. En la Figura 1 se indica su patrón de difracción y se marcan los picos de los cuatro compuestos detectados: (a) el compuesto ternario $\mathrm{Fe}_{6} \mathrm{Sn}_{6} \mathrm{Zr}$, observado también por Savidan y col. [4] a $900^{\circ} \mathrm{C}$ y por Rebellato y col. [8] a 
$800^{\circ} \mathrm{C}$ en una aleación diferente; (b) el compuesto binario $\mathrm{Fe}_{5} \mathrm{Sn}_{3}$ reportado como existente en el binario Fe$\mathrm{Sn}$, pero a partir de $806^{\circ} \mathrm{C}$ [9]; (c) el $\beta \mathrm{Sn}$; y (d) un pico que correspondería a la fase $\mathrm{FeSn}_{2}$. Los tres primeros corresponderían a las fases mayoritarias, habiendo evidencia metalográfica de este hecho. Con técnicas de MAE se midieron las composiciones de las tres fases mayoritarias (Tabla 2). También debemos señalar que con MAE se midió la composición de puntos correspondientes a una fase de estequiometría $\mathrm{Fe}_{92,8} \mathrm{Sn}_{7,2}$, que no está reportada en el diagrama Fe-Sn, así como escasos puntos correspondientes al compuesto Y [4-8]. Dado que en esta región del diagrama sólo puede haber un máximo de tres fases en equilibrio (regla de las fases de Gibbs), los compuestos $\mathrm{FeSn}_{2}, \mathrm{Fe}_{92,8} \mathrm{Sn}_{7,2}$ y Y (observadas metalográficamente los dos últimos en escasa cantidad) se consideran inestables, ya sea por ser fases retenidas desde la fundición o bien por ser por resultantes de la complejidad de reacciones que se producen por la presencia de un líquido en la aleación ( $\mathrm{Sn}$ a $\left.800^{\circ} \mathrm{C}\right)$.

La muestra T2 TT resultó claramente ubicada en un campo de tres fases: $\mathrm{ZrFe}_{2}(\mathrm{C} 15)$, (C36) y N (Figura 2). Las tres fases fueron identificadas por su estructura cristalina con DRX y composición química con EDS y MAE (Tabla 2).

La muestra T3 TT, luego del tratamiento térmico y posterior templado se redujo rápidamente a polvo resultando imposible su medición con EDS y MAE. Una degradación similar fue observada por Nieva y Arias en muestras de composición cercana tratadas a 800 y $900^{\circ} \mathrm{C}$ [3]. Sólo se obtuvo información de esta muestra con DRX. Las fases cristalinas identificadas fueron las ortorrómbicas $\mathrm{N}$ y $\mathrm{ZrSn}_{2}$ y la fase X”. Los picos de esta última fueron obtenidos por comparación con lo observado en la misma fase por Savidan y col. [4] a $900^{\circ} \mathrm{C}$

La muestra T4 TT presentó a la fase X" como principal. Además, se identificaron con DRX y EDS las fases $\mathrm{N}$ y $\mathrm{ZrSn}_{2}$ (Figura 3). A pesar de estar en el mismo triángulo de compatibilidad de la muestra T3, la T4 no sufrió la misma degradación. La mayor presencia de la fase $\mathrm{ZrSn}_{2}$ en T3 sería la fuente de inestabilidad en su integridad física.

La muestra T5 TT presentó a la fase $\mathrm{ZrFe}_{2}(\mathrm{C} 15)$ como principal. En menor medida se identificó con la fase (C36), de simetría hexagonal, con parámetros de red $a=b=4,982 \AA$, $c=16,207 \AA$ y composición química $\mathrm{Fe}=69,1 \%, \mathrm{Sn}=14 \%, \mathrm{Zr}=16,9 \%$, en \% atómicos.

La muestra T6 TT presentó a la fase (C36) como principal. En menor medida se identificó al $\alpha$ Fe, simetría cúbica, con parámetros de red $\mathrm{a}=\mathrm{b}=\mathrm{c}=2,878 \AA$ y composición química $\mathrm{Fe}=97,1 \%, \mathrm{Sn}=2,4 \%, \mathrm{Zr}=0,5 \%$, en \% atómicos.

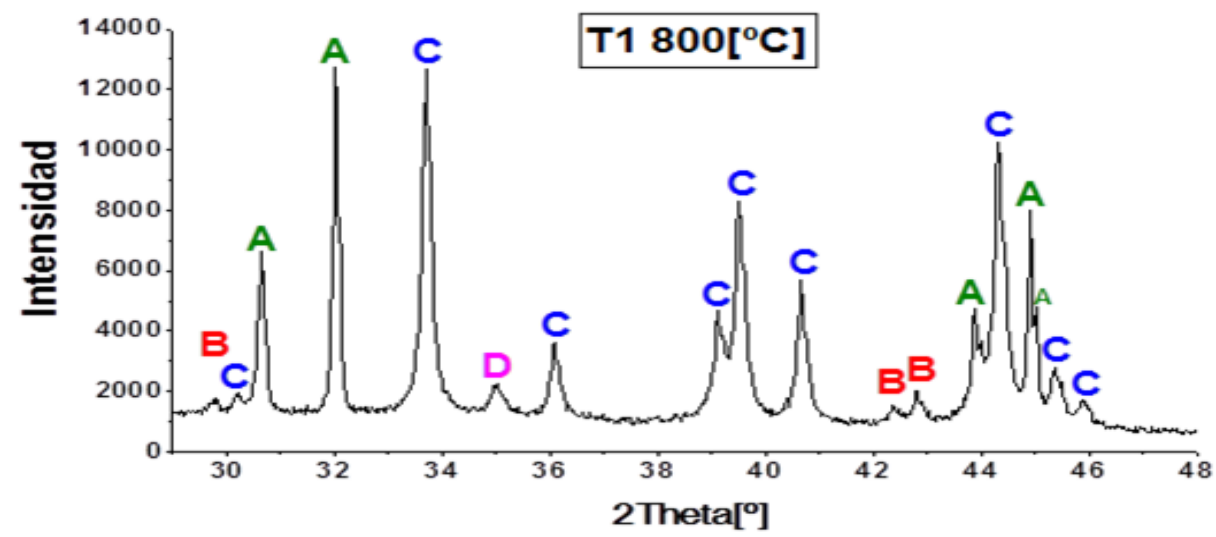

Figura 1: Patrón de DRX de T1 TT a $800^{\circ} \mathrm{C}$. Fases identificadas: (A) $\beta \mathrm{Sn}$, (B) $\mathrm{Fe}_{5} \mathrm{Sn}_{3}$, (C) $\mathrm{Fe}_{6} \mathrm{Sn}_{6} \mathrm{Zr}$, (D) FeSn . 


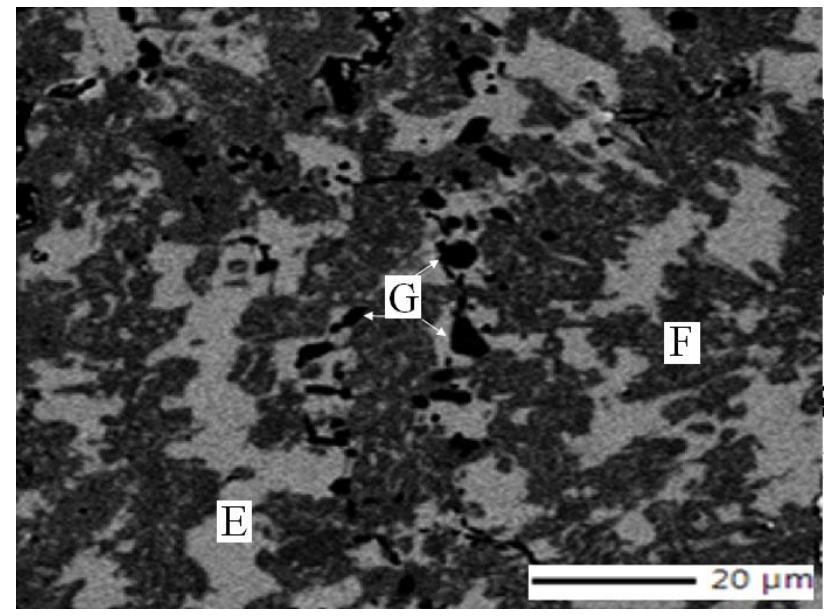

Figura 2: Muestra T2 TT a $800^{\circ} \mathrm{C}$. Imagen con MEB, electrones retrodifundidos. Fases identificadas: (E) Fase N, (F) (C36), (G) $\mathrm{ZrFe}_{2}(\mathrm{C} 15)$.

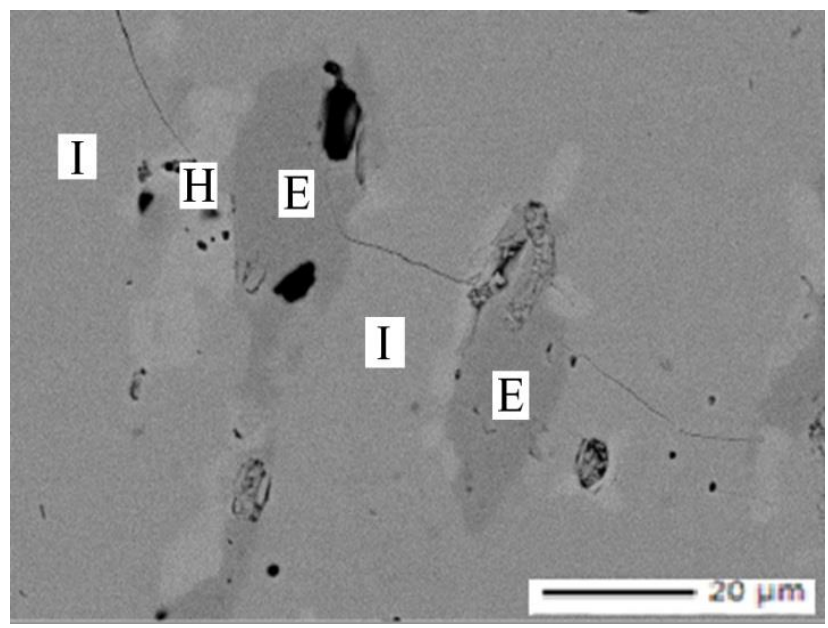

Figura 3: Muestra T4 TT a $800^{\circ} \mathrm{C}$. Imagen con MEB, electrones retrodifundidos. Fases identificadas: (E) Fase N, (H) $\mathrm{ZrSn}_{2}$, (I) Fase X".

\section{CONCLUSIONES}

De acuerdo a los datos experimentales recabados en el presente trabajo y a partir del diagrama de fases Fe$\mathrm{Sn}-\mathrm{Zr}$ a $800^{\circ}$ propuesto por Nieva y Arias [ 3 ] y ampliado por Rebellato y col. en [8] se trazan los límites de equilibrio de fases en las regiones bajo estudio en la Figura 4.

En este diagrama se incluyen los compuestos encontrados a esta temperatura en este trabajo: $\mathrm{Fe}_{5} \mathrm{Sn}_{3} /$ $\mathrm{Fe}_{6} \mathrm{Sn}_{6} \mathrm{Zr} / \mathrm{Y} / \mathrm{ZrFe}_{2}(\mathrm{C} 15)$ / (C36) / N. Se verificó e incluyó además la presencia de la fase X" [4]. Se determinaron campos de existencia de 1, 2 y 3 fases. Se trazaron los límites de equilibrio de fases en las regiones bajo estudio, completando en gran proporción el diagrama de fases para el corte isotérmico analizado.

En el diagrama de fases de la Figura 4 se dibujan sombreados los triángulos de compatibilidad informados en este trabajo. Se marcan con línea llena los límites de existencia de fase previamente reportados y con línea de trazos los límites probables.

\section{AGRADECIMIENTOS}

Este trabajo fue apoyado por el Consejo de Investigaciones de la Universidad Nacional de Tucumán (CIUNT) a través del Proyecto 26/E526 y con una Beca CIUNT. Agradecemos al Dr.Daniel Vega del CACCNEA por los resultados de DRX, al CIME-Tucumán por las mediciones con SEM-EDS, al Dr. Rubén González y los Sres. Lucas Pascual y Rodrigo Ramelli del CAC-CNEA por las mediciones con EPMA y al BUF Horacio Brizuela del LAFISO por su apoyo y colaboración desde inicio del trabajo. 


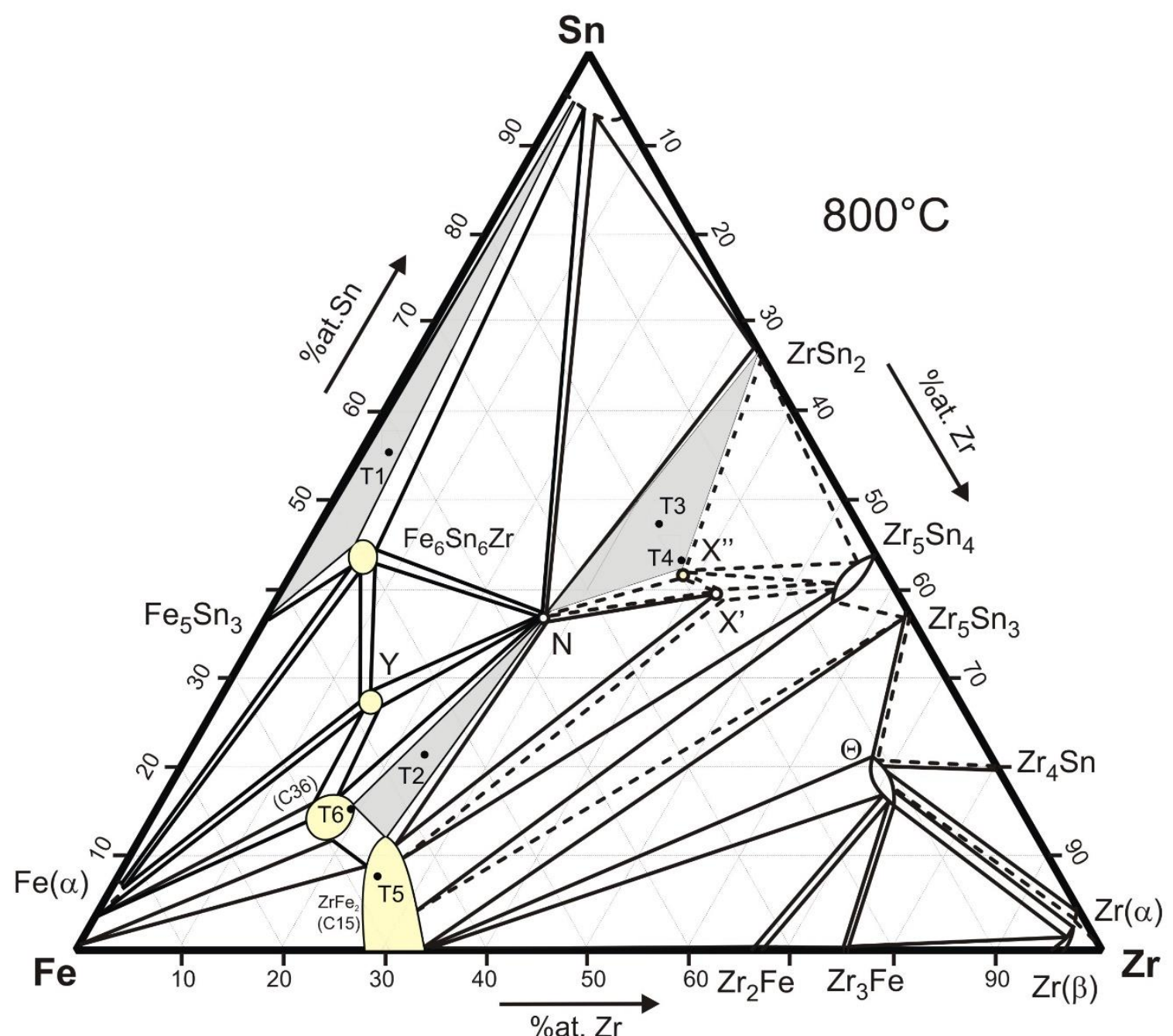

Figura 4: Diagrama de fases $\mathrm{Fe}-\mathrm{Sn}-\mathrm{Zr}$ propuesto para el corte isotérmico de $800^{\circ} \mathrm{C}$. Límites de fases: (一) medidos, (---) probables. $(\bullet)$ Composiciones nominales de las aleaciones analizadas.

\section{BIBLIOGRAFÍA}

[1] LIU, S., DU, Y., XU, H., HUANG, B., "Iron-Tin-Zirconium" In: Ternary Alloy Systems, Phase Diagrams, Crystallographic and Thermodynamic Data, Effenberg G., Ilyenko S. Editors. Berlin, Heidelberg, New York: Springer-Verlag, pp. 480-492, 2009.

[2] NIEVA, N., ARIAS, D., "A new ternary compound in the Zr-Sn-Fe system", Journal of Nuclear Materials, v. 277, n. 1, pp. 120-122, Jan. 2000.

[3] NIEVA, N., ARIAS, D., "Experimental partial phase diagram of the Zr-Sn-Fe system", Journal of Nuclear Materials, v. 359, n. 1-2, pp. 29-40, Dec. 2006.

[4] SAVIDAN, J. C., JOUBERT, J. M., TOFFOLON-MASCLET, C., "An experimental study of the Fe-Sn$\mathrm{Zr}$ ternary system at $900{ }^{\circ} \mathrm{C}$ ", Intermetallics, v. 18, n. 11, pp. 2224-2228, Nov. 2010.

[5] RAGHAVAN, V., "Fe-Sn-Zr (Iron-Tin-Zirconium)", Journal of Phase Equilibria and Difussion, v. 32, n. 4, pp. 379-380, Aug. 2011.

[6] NIEVA, N., ARIAS, D., "New results in the experimental study of the Zr-Sn-Fe phase diagram. 800 and $900^{\circ} \mathrm{C}$ isothermal sections", PI-12, International Conference on Phase Diagrams Calculations and Computational Thermochemistry, CALPHAD XXXIII, Krakow, Poland, May 2004.

[7] NIEVA, N., JIMENEZ, M.J., GOMEZ, A., CORVALAN MOYA, C., ARIAS, D., "Diagrama de fases feSn-Zr. Nuevos resultados experimentales", In: Anales 12 Congreso Binacional de Metalurgia y Materiales, CONAMET/SAM 2012, Ed. CD, Valparaíso, Chile, Oct. 2012. 
[8] REBELlATO, F., CORVALAN, C., ARIAS, D., NIEVA, N., "Nuevos resultados experimentales para el diagrama de fases Fe-Sn-Zr. Región central y adyacente al binario Fe-Sn”, In: Congreso Internacional de Metalurgia y Materiales, XIII IBEROMAT, 14॰ SAM/CONAMET, XIII, Simposio MATERIA, Ed. CD, Santa Fe, R. Argentina, Oct. 2014.

[9] OKAMOTO, H., "Fe-Sn (Iron-Tin)" In: Phase Diagrams of Binary Iron Alloys, ASM International, Materials Park, OH, pp. 385-392, 1993. 